\title{
Fixed-target program upgrade and prospects at $\mathrm{LHCb}$
}

\author{
Edoardo Franzoso ${ }^{1,2, *}$ for the LHCb Collaboration \\ ${ }^{1}$ Università degli Studi di Ferrara, Ferrara, Italy \\ ${ }^{2}$ INFN Sezione di Ferrara, Ferrara, Italy
}

\begin{abstract}
LHCb}$ has the unique capability to operate in fixed-target mode to study collisions of the LHC beams on fixed targets. In Run3, the internal gas target is going to be upgraded to allow for a wider selection of target gas species and a significant increase of the rates of fixed-target collisions by up to two orders of magnitude. Along with significant data acquisition and tracking upgrades, the SMOG2 system greatly enhances the reach of LHCb's heavy ion program.
\end{abstract}

\section{Fixed-target collisions in LHCb}

The LHCb detector was originally conceived and designed to study heavy-flavour physics. However, today, its research program spans over a wide spectrum of high-energy physics phenomena and LHCb acts effectively as a general-purpose experiment in the forward direction with excellent tracking and particle identification performance. Since the installation of SMOG (System for Measuring overlap with gas) in 2015, LHCb offers the unique possibility to study fixed-target collisions in addition to the standard collider operations.

The SMOG system was originally designed to inject noble gases inside the primary LHC vacuum in correspondence of the LHCb vertex locator (VELO) for precise luminosity determinations through the beam-gas imaging technique [1]. The gas was injected using a Gas Feed System reaching a local pressure of $2 \times 10^{-7}$ mbar. During the LHC RUN 2, the LHCb fixed-target program included runs with different targets and beam energies: between 2015 and 2018, SMOG has collected data with proton beams colliding on $\mathrm{He}, \mathrm{Ar}$ and $\mathrm{Ne}$; additionally, in 2018, data with $\mathrm{Pb}$ beams on a $\mathrm{Ne}$ target have been recorded.

The fixed-target configuration in LHCb opens the possibilities to perform measurements of great interest for cosmic rays and heavy-ion physics with centre-of-mass energies from 40 to $115 \mathrm{GeV}$, corresponding to beam energies ranging from 0.9 to $7 \mathrm{TeV}$. Furthermore, the forward acceptance of the LHCb spectrometer $(2<\eta<5)$ is perfectly suited to cope with the strong boost in the lab frame of the final-state particles produced in fixed-target collisions. In the center of mass frame, particles produced at central and backward rapidities are observed, offering the possibility to study the high Bjorken-x region in the target nucleons or nuclei (large Bjorken-x can be accessed in the collider mode at much higher values of $q^{2}$, the squared transferred four-momentum.)

\footnotetext{
*e-mail: edoardo.franzoso@cern.ch
} 


\subsection{Physics results from fixed-target measurements in RUN2}

During the RUN 2, LHCb performed the first fixed-target measurements with the SMOG setup. Charm production studies in samples of pAr at $\sqrt{s}=86 \mathrm{GeV}$ and $\mathrm{pHe}$ at $\sqrt{s}=110$ $\mathrm{GeV}$ have been published [2], reporting no evidence of strong intrinsic charm contribution. Moreover, the $c \bar{c}$ cross-section has been measured on pHe data very precisely in an unexplored energy region. Another interesting result is related to the secondary production of antiprotons in collisions of cosmic rays with the interstellar medium. Specifically, LHCb measured the antiproton production cross-section in the 10-100 GeV energy range [3] using a $6.5 \mathrm{TeV}$ proton beam colliding on a He target, one of the main constituents of the interstellar medium. Only antiprotons originating directly from pHe collisions and decays of resonances via strong interactions were considered in the analysis. The experimental uncertainties are small compared to the spread of theoretical predictions and the results obtained are relevant for dark matter annihilation studies, and contributed to reduce the tension between the theoretical predictions and the measurements by the PAMELA and AMS-02 experiments [4].

Although originally not conceived for physics measurements, during RUN 2 SMOG has successfully demonstrated the capabilities of LHCb as a fixed-target experiment. There are, however, some drawbacks. One of those is the diffusion of the injected gas along a \pm 20 $\mathrm{m}$ long sector of the LHC beam pipe. As a consequence, simultaneous data taking with $\mathrm{pp}$ collisions was not possible due to the overlapping of the luminous region and SMOG could only collect data during short dedicated runs. Additionally, the injected gas density could not be measured precisely and the only way to infer the luminosity was by exploiting the cross section of known processes, such as the single-electron scattering [3]. To overcome these constraints, an upgrade of the SMOG system is undergoing during the Long Shutdown 2 of LHC.

\section{The upgrade of the fixed-target system}

In general, the fixed-target program will profit from all the improvements in the detector performance expected in RUN 3. In particular, the SMOG system is undergoing a specific upgrade through the installation of a gas storage cell upstream the pp interaction point. The new system [5], called SMOG2, is shown in figure 1a.

The storage cell offers the opportunity to have a controlled environment designed specifically for a fixed-target experiment along with the possibility to inject several gas species in addition to those already available with SMOG. In particular, also $\mathrm{H}_{2}, \mathrm{D}_{2}, \mathrm{~N}_{2}, \mathrm{O}_{2}, \mathrm{Kr}$ and $\mathrm{Xe}$ can in principle be injected in addition to $\mathrm{He}, \mathrm{Ne}$ and $\mathrm{Ar}$. SMOG2 is also coupled with a new Gas Feed System able to provide a precise density measurement of the target, hence a precise luminosity determination (not possible with the previous system). Another key point is the possibility to have a well-defined interaction region upstream of the pp interaction point (figure 1b), this feature allows easy separation of the two types of interactions related to beam-beam and beam-target collisions, respectively. A high reconstruction efficiency is expected in the fiducial volume of the cell despite the upstream position of the beam-gas primary vertices compared to the nominal collider interaction point. The possibility to run in parallel with the collider mode and to simultaneously collect beam-beam and beam-gas data for the RUN 3 can be considered since preliminary studies confirm that the performance of the measurements in the collider mode will not be affected. The last major improvement is the increase of target areal density by up to two order of magnitude, meaning a higher luminosity for fixed-target events, by assuming the same gas flow provided for SMOG. 


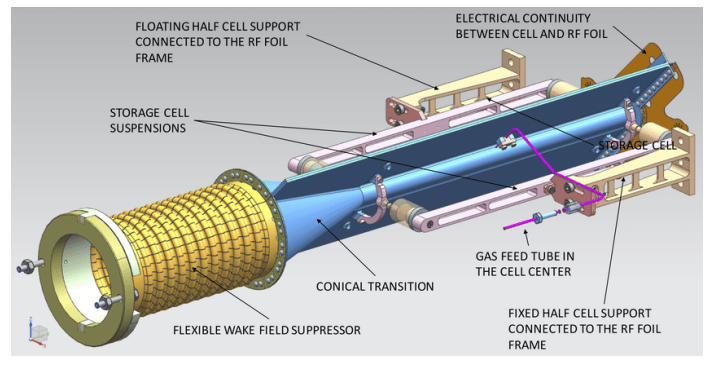

(a)

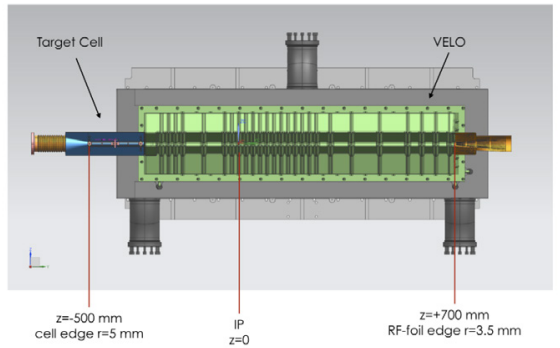

(b)

Figure 1. (a) Design of the SMOG2 storage cell; (b) side view of the storage cell connected to the VELO

Table 1. Integrated luminosity and yields projection for SMOG2 in RUN3 compared with the largest SMOG sample available from RUN2

\begin{tabular}{lll}
\hline & SMOG pNe $(\sqrt{s}=68 \mathrm{GeV})$ & SMOG2 pAr $(\sqrt{s}=115 \mathrm{GeV})$ \\
\hline Integrated luminosity & $\sim 100 \mathrm{nb}^{-1}$ & $\sim 100 \mathrm{pb}^{-1}$ \\
syst. error on $J / \Psi \mathrm{x}-\mathrm{sec}$. & $6-7 \%$ & $2-3 \%$ \\
$J / \Psi$ yield & $15 \mathrm{k}$ & $35 \mathrm{M}$ \\
$D^{0}$ & $100 \mathrm{k}$ & $350 \mathrm{M}$ \\
$\Lambda_{c}$ yield & $1 \mathrm{k}$ & $3.5 \mathrm{M}$ \\
$\Psi(2 S)$ yield & 150 & $400 \mathrm{k}$ \\
$\Upsilon(1 S)$ & 4 & $15 \mathrm{k}$ \\
Low-mass $\left(5<M_{\mu \mu}<9 \mathrm{GeV} / \mathrm{c}^{2}\right)$ Drell-Yan yield & 5 & $20 \mathrm{k}$
\end{tabular}

\section{Physics Prospects for RUN 3}

The physics opportunities offered by the fixed-target program at LHCb are unique. The fixedtarget configuration instrumented with the SMOG2 storage cell will significantly extend the studies already performed with SMOG [6]. In Table 1, a projection of the yields expected for SMOG2 is reported as compared to those measured with SMOG. Charm measurements, which are vital for studies of cold nuclear matter effects and heavy-ion physics, will benefit from the increased statistics and better luminosity determination with SMOG2. Moreover, the possibility to measure beauty production in $\mathrm{pA}$ collisions can be considered since a significant yield of reconstructed $\Upsilon(1 S)$ is expected (Tab. 1). Also QGP-related flow observables and correlations can be studied in the full centrality range in $\mathrm{PbA}$ collisions.

Cosmic rays studies will highly profit from the opportunity to inject hydrogen and deuterium with the possibility to extend the study of antiproton production with the full centre of mass range available and to measure the ratios $\sigma(p H e \rightarrow \bar{p} X) / \sigma(p H \rightarrow \bar{p} X)$ and $\sigma(p D \rightarrow \bar{p} X) / \sigma(p H \rightarrow \bar{p} X)$. A deuterium target will also allow testing isospin symmetry effects. The fixed-target mode would probe the energy scale that is expected to be most relevant for the production of light anti-nuclei such as $\bar{d}$ so the opportunity to detect light anti-nuclei in $\mathrm{LHCb}$ is under investigation.

Looking at the nucleon structure studies, SMOG2 will be able to exploit the increased statistics to probe quark and gluon PDFs in nucleons and nuclei, especially at high-x and moderately-high $q^{2}$. Drell-Yan and weak-boson production are suitable to constraint theoretical models and reduce uncertainties on the light quark and anti-quark PDFs. Furthermore, heavy-flavour production, which is dominated by gluon-gluon interactions in high-energy hadronic collisions, will allow accessing the poorly known gluon distribution functions. 


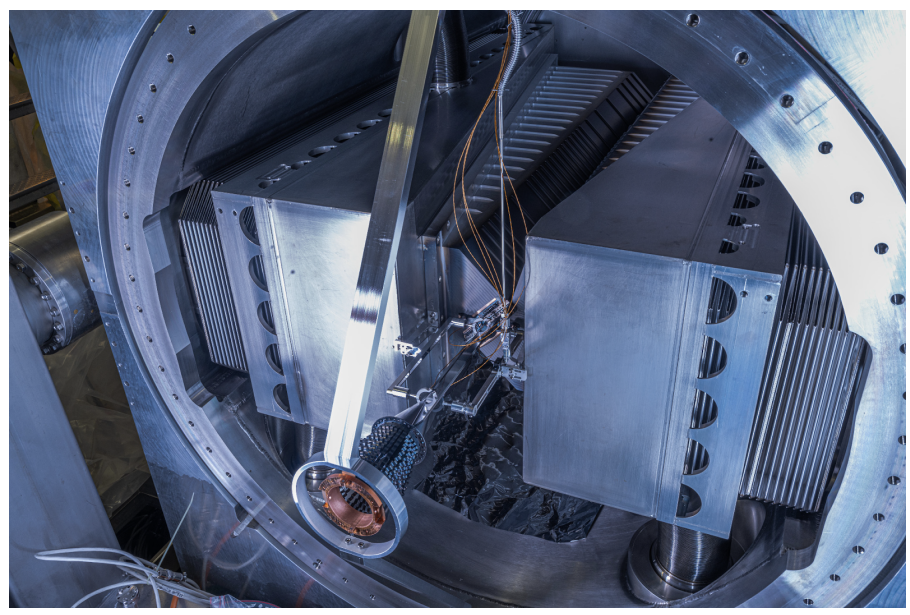

Figure 2. Storage cell installed and connected to the VELO vessel

\section{Conclusions}

$\mathrm{LHCb}$ is, as today, the only existing experiment capable to run both in fixed-target and collider mode and will continue a pioneering fixed-target program with SMOG2. The storage cell has been installed in front of the VELO as can be seen in figure 2, meanwhile, the new Gas Feed System will be ready by the end of 2021. SMOG2 will provide a wide choice of targets in combination with proton or ion beams and a significantly higher luminosity compared to SMOG, extending the LHCb fixed-target program physics reach.

\section{References}

[1] C. Barschel, Precision luminosity measurements at LHCb with beam-gas imaging. CERN-THESIS-2013-301, RWTH Aachen University (2014)

[2] LHCb collaboration, R. Aaij et al., First measurement of charm production in its fixedtarget configuration at the LHC. Phys. Rev. Lett. 122, 132002 (2019).

[3] LHCb collaboration, R. Aaij et al., Measurement of antiproton production in pHe collisions at $\sqrt{s}=110 \mathrm{GeV}$. Phys. Rev. Lett. 121, 222001 (2018).

[4] Boudaud, Mathieu, et al., AMS-02 antiprotons consistency with a secondary astrophysical origin. Phys. Rev. Res. 2.2, (2020).

[5] LHCb collaboration SMOG2, Technical Design Report (2018).

[6] PBC Report, Physics opportunities with the fixed target program of the LHCb experiment using an unpolarized gas target. LHCb-PUB-2018-015 (2018). 